\section{La experiencia de ser un 'niño débil y enfermo' lejos de su hogar: el caso del Asilo Marítimo, Mar del Plata (1893-1920)*}

\section{The experience of a 'weak and sick childhood' far from home: the experience of the Maritime Asylum, Mar del Plata (1893-1920)}

ÁLVAREZ, Adriana. La experiencia de ser un 'niño débil y enfermo' lejos de su hogar: el caso del Asilo Marítimo, Mar del Plata, 1893-1920. História, Ciências, Saúde - Manguinhos, Rio de Janeiro, v.17, n.1, jan.-mar. 2010, p.13-31.

\section{Resumen}

Este trabajo tiene como objetivo analizar la vida cotidiana de los 'niños tuberculosos', provenientes de la ciudad de Buenos Aires, que fueron internados en el Hospital Marítimo, ubicado a $400 \mathrm{~km}$ de la metrópolis, en la localidad marítima de Mar del Plata. En 1893, surgió la idea en la Sociedad de Beneficencia de la Capital Federal de fundar un hospital y el Asilo Marítimo destinado a niños enfermos de tuberculosis ósea en general, a niños débiles y convalecientes y también al tratamiento de pacientes con tuberculosis extra-pulmonar. En este artículo, se pretende avanzar en la comprensión de dos problemáticas vinculadas entre sí: por un lado las características de la ingerencia institucionalizada de la Sociedad de Beneficencia y por el otro, la experiencia que estos niños tuberculosos vivieron en ese ámbito. Palabras claves: niñez; tuberculosis; asilo; historia; Argentina.

\section{Abstract}

The objective of this work is to analyze the daily life of 'tubercular children'” coming from the city of Buenos Aires. They were hospitalized in the Maritime Asylum located in the seaside city of Mar del Plata, 400 kilometers from the capital. In 1893, in the Charitable Society of the Federal Capital, the idea arose of founding a hospital and maritime asylum for children ill with osseous tuberculosis in general, weak and convalescing children and the treatment of patients with extra-pulmonary tuberculosis. This paper attempts to advance the understanding of two inter-related topics: on the one hand, the characteristics of the institutionalized meddling of the Charitable Society, and, on the other, the experience that these tubercular children had in this institution.

Keywords: childhood; tuberculosis; asylum; history; Argentina. 
$\mathrm{L}$ as concepciones acerca de la relación entre la infancia y la enfermedad han cambiado considerablemente a lo largo de la historia. Por tal motivo, al enfocar la infancia enferma es ineludible observar las distintas conceptualizaciones que en el período estudiado se elaboraron. Ello se debe a que la manera de entender la infancia y las pestes tiene que ver con los modos de organización socioeconómica de las sociedades, los intereses sociopolíticos y el desarrollo de las teorías científicas, tanto en el campo médico como pedagógico. Por consiguiente, se trata de un concepto cuyo dinamismo depende del ritmo de los acontecimientos históricos que lo cruzan y de un discurso que aparenta ser unívoco, pero que en la práctica muestra una diversidad que este trabajo se esfuerza por mostrar.

Por lo tanto, el desafío de este ensayo es enfocar en forma conjunta la comprensión tanto del universo infantil como el de la enfermedad, no como divisiones estancas sino reconociendo dinamismos propios pero también compartidos. Comprendiendo que si bien no encontraremos en la conjunción de estas categorías un devenir paralelo y armónico, el desafío consiste en explicar las conexiones entre ambas que generaron determinadas formas de concebir las enfermedades infantiles, las que estuvieron motorizadas tanto por necesidades políticas, ideológicas y sociales como por el influjo que jugaron los médicos y la medicina en los destinos del Estado y en el diseño de políticas sociales.

Este trabajo se ubica en la Argentina de fines del siglo XIX y principios del XX, momento que coincide con el surgimiento de un concepto moderno de niñez lo que era tributario de una noción de infancia cualitativamente distinta a la de las etapas anteriores. Este proceso, que algunos autores denominan 'infantilización de la niñez', implicó poner en marcha un proceso a través del cual la sociedad comenzó a proteger a los niños visualizando en las instituciones médicas y escolares las bases sobre las cuales moldear a estos futuros trabajadores y ciudadanos.

Philippe Ariès sentó las bases de este tipo de estudios en el año de 1960. La tesis central del historiador francés es muy conocida y ha generado una amplia gama de estudios, pero también de controversias. El texto de Ariès (1987) sostiene que durante la Edad Media no existió un concepto definido de niñez, en la medida en que no existía un espacio simbólico reservado a los niños, lo que fue revertido con la llegada de la modernidad. Con el transcurrir del tiempo, son varios los investigadores que se han encargado de cuestionar, matizar y de ampliar algunas de las cuestiones centrales a esa tesis. En relación a los intereses de este ensayo en particular, algunas obras resultan elementales para la interpretación de las características que asumió la internación de los niños tuberculosos y de los 'débiles' o 'pretuberculosos' en el Sanatorio Marítimo.

En tal sentido, nos aproximamos al surgimiento del concepto moderno de niñez, entendiéndolo como un conjunto de mutaciones que no deben ser interpretadas como cambios aislados sino elementos de un nuevo sistema global de referencias que comprende no solo ideas nuevas, sino también nuevas relaciones sociales y nuevas instituciones. ${ }^{1}$ Stone (1986) introduce una visión centrada en la existencia de un concepto de infancia en otras culturas distintas a la occidental, donde predomina esa idea de mutación más que de ruptura entre las distintas épocas. Otros textos de los cuales este ensayo se nutre es el de L. Pollock (1983) quien sostiene que los padres han cuidado y amado a sus hijos a lo largo de toda la historia, problemática que es retomada en este trabajo pero vinculándola al rol 
que cumplieron en relación a la aceptación o no del tratamiento médico brindado a sus hijos. Los textos mencionados son tributarios de la tendencia que dominó a la producción historiográfica en los años 1980, la que a diferencia de la década del 1960 - momento en cual destelló la tesis de P. Ariés - se ocupó de marcar los límites de la modernidad basándose en cuestionar la confianza que sobre la noción de progreso reinaba entre los historiadores coetáneos al historiador francés.

Por lo tanto, entendemos que es necesario rescatar las especificidades de cada proceso, entendiendo cómo Buenos Aires construyó su propia visión de la niñez enferma, destacando en esto el rol que cumplió el proceso de modernidad que alentó la emergencia de una noción cualitativamente distinta a la de las etapas precedentes. En tal sentido, el objetivo de este trabajo es aproximarse a ese proceso, pero desde un enfoque acotado al niño tuberculoso y pre-tuberculoso ${ }^{2}$ perteneciente a los sectores populares. La razón de este recorte obedece a que reconstruir la historia de la niñez acarrea de por sí una gran complejidad reiteradamente señalada por los especialistas, entre otras cuestiones, debido a la dificultad en torno a las fuentes documentales. Pero se complejiza aún más si lo acotamos a una situación determinada, como es la enfermedad. Por ello, la estrategia metodológica consiste en realizar un enfoque micro institucional, que se justifica por la relevancia del establecimiento escogido, obra de la Sociedad de Beneficencia de la Capital Federal, principal dador de servicios sanitarios para la época. Y también por la disponibilidad de fuentes existentes en el repositorio del Archivo General de la Nación (AGN). Por lo expuesto, y entendiendo que los registros históricos específicos que se refieren a los niños tuberculosos son escasos y vagos, desde esta mirada entendemos que se puede contribuir a brindar un primer panorama susceptible de ser comparado con otros procesos similares de manera de acercarnos a explicaciones más amplias y complejas.

El corte cronológico obedece a que en 1893 se inauguró el Asilo y Sanatorio Marítimo a $400 \mathrm{Km}$ de la ciudad de Buenos Aires, momento que coincide con cambios en las concepciones acerca de la cuestión de la niñez en la Argentina. La frontera de la década del 1920 tiene que ver con las transformaciones internas que experimentó el establecimiento y que afectaron directamente el modo de cómo se venía instrumentado la institucionalización de estos niños pertenecientes a los sectores más pobres de la sociedad porteña.

Algunas de las cuestiones que cruzan esta ponencia tienen que ver con los vacíos existentes en el contexto historiográfico argentino ${ }^{3}$ del cual se hará una breve referencia a los efectos de enmarcar las preguntas a responder.

\section{Contexto historiográfico}

Entre fines del siglo XIX y mediados del siglo XX, una de las enfermedades que provocó el mayor índice de mortalidad en la Argentina fue la tuberculosis. Esta cuestión fue motivo de interés académico. Así, los primeros trabajos de Diego Armus abordaban la tuberculosis en el territorio de la Argentina como un problema social. A partir de allí, numerosos estudios realizados por el mismo historiador dan cuenta de la enfermedad en relación a la profesionalización médica, al desarrollo de la tuberculosis en determinadas ocupaciones laborales y en las historias de los propios enfermos que protestan por el abandono por 
parte del Estado (Armus, 1966, 2000b, 2005). En los últimos años, en Argentina, nuevos grupos de investigación han producido estudios históricos sobre la relación entre la salud y la enfermedad, instituciones sanitarias - públicas y privadas - y políticas de Estado, focalizados en el interior del territorio nacional, como ser Córdoba, La Pampa, Jujuy y Tucumán, entre otras (Carbonetti, 2004). En cuanto a los trabajos cuyo eje es la relación salud/enfermedad/niñez, los estudios académicos giran en torno al problema de la puericultura: protección de la madre y el niño, las instituciones públicas de salud de Buenos Aires para la protección del niño, entre otros temas. ${ }^{4}$ De este modo, nuestro trabajo resulta novedoso en cuanto a que el Hospital Marítimo y el Solarium se especializaron en la atención de niños tuberculosos y pre tuberculosos, en su mayoría derivados de los hospitales de la ciudad de Buenos Aires a cargo de la Sociedad de Beneficencia de la Capital ${ }^{5}$, lo que lo convertía en una extraña mezcla de sanatorio y colonia de verano.

En este sentido, la producción historiográfica argentina cuenta con algunas investigaciones - no muchas - que explican el funcionamiento de las colonias y las escuelas destinadas a los niños débiles, mas no a los sanatorios propiamente dichos. En La ciudad impura, Diego Armus (2007, p.101) sostiene: "Las colonias y escuelas para niños débiles eran la materialización de una de las tantas visiones civilizadoras que estaban reaccionando frente al complejo problema de la morbilidad y mortalidad infantil ...". Lo que implicó una reacción destinada a revertir las consecuencias del mal vivir "que debía ser afrontada desde cada uno de los hogares o por fuera de ellos a través de Instituciones del Estado o la Sociedad Civil" (p.101). Por otra parte, Di Liscia (2005) muestra el funcionamiento de estas instituciones en un ámbito no capitalino como era el territorio nacional de La Pampa, donde se detiene en las particularidades y las diferencias que allí adquirió dicho proceso histórico. Desde una perspectiva algo diferente, Gustavo Vallejos (2007) entiende estas colonias como parte del corpus con el que la biotipología de la época intervino en el cuerpo social buscando una regeneración extraurbana, razón por la cual focaliza su estudio en la colonia de niños débiles de Necochea, de la provincia de Buenos Aires. Por último, la investigación de Lucia Lionetti (2008) se centra en las escuelas para niños débiles en el centro de la provincia de Buenos Aires, Tandil. Aunque el énfasis está puesto en la cuestión pedagógica, la autora dialoga de manera permanente con las cuestiones médico asistenciales de la época.

Del paneo historiográfico realizado, se observa que en la producción nacional no abundan los enfoques 'desde adentro' sobre instituciones abocadas al cuidado de la niñez enferma, como el que se propone analizar en este artículo, dado que las colonias como las escuelas para niños débiles procuran prevenir el desarrollo de la enfermedad mas no el tratamiento de la misma como sucedió en el Sanatorio Marítimo. Tampoco son abundantes estudios que se centren en la tuberculosis infantil, excepto lo analizado por Diego Armus (2007) en el capitulo "La forja del cuerpo sano: niñez, educación física, fútbol y tuberculosis" de su libro La ciudad impura, donde muestra que por temor al contagio y en pos de las acciones preventivas se impulsaron la gimnasia respiratoria y antituberculosa y se estimularon algunos deportes. De la mano de los deportes, el autor ingresa en la problemática de la tuberculosis infantil, analizando una serie de iniciativas institucionales cuyos resultados son interpretados como 'limitados' puesto que nunca lograron 
transformarse en una alternativa realmente masiva. Este libro, como otros trabajos del mismo autor, ha servido de disparador de una serie de interrogantes vinculadas a cuestionarnos acerca de la dinámica institucional de los establecimientos dedicados al tratamiento de la tuberculosis infantil, como su permeabilidad o no a los postulados médicos en boga por entonces, entre otras preguntas.

A pesar de los vacíos antes señalados, la problemática de la niñez en la Argentina ha sido abordada desde enfoques diversos como es el de la mortalidad (Mazzeo, 1993), el mundo del trabajo (Suriano, 1990; Rustán, Carbonetti, 2000), el discurso maternal (Nari, 2004; Barrancos, 1999, 2001; Lobato, 2000), entre otros, o también a partir del abandono (Guy, 1994; Moreno, 2000; González, 2000), producciones que nutren esta investigación como también lo hacen las referidas a las prácticas benéficas (Bonaudo, 2006) y las centradas en la Sociedad de Beneficencia de la capital (Guy, 2000; Pita, 2004, 2007), entre otras.

Sin embargo, no existe un enfoque que integre las variables de mortalidad, enfermedad y condiciones materiales de existencia y niñez que eche luz sobre los dispositivos institucionales, los discursos, las diversas formas de padecer las enfermedades y el impacto de los males según estratos sociales, como también las respuestas sociales que frente a las primeras intervenciones se dieron. No se trata de preguntarnos sobre los dispositivos ordenadores montados con el fin de prevenir el 'mal nacer' o 'el mal vivir', sino de explicar qué se hizo con la niñez enferma, qué se entendía por niño enfermo y qué abanico de cuestiones abarcó el discurso médico y político cuando se referían a ello. En definitiva, nos preocupa entender cómo se fueron gestando las políticas de sanidad infantil en nuestro país.

En esta oportunidad, nos preguntamos sobre las formas institucionales que se le dieron a la niñez popular tuberculosa como una forma de describir y de explicar las diversas formas de enfermar y de curar, la difusión del contagio, como la heterogeneidad de situaciones dentro de un mismo espacio institucional, como también verificar la vigencia o no del discurso hegemónico que en torno a la niñez pobre se gestó en los ámbitos de poder.

Para ello, a continuación se presentará el universo social del cual provenían los niños que fueron internados en el Asilo Marítimo.

\section{La situación de la niñez en Buenos Aires a fines del siglo XIX y principios del XX: el caso de los sectores populares}

Buenos Aires, a fines del siglo XIX, atravesaba un acelerado proceso de transformación vinculado con la presencia masiva de inmigrantes extranjeros que llegaban a sus puertos procedentes, principalmente, de las costas europeas. Este cambio en el mapa poblacional trajo aparejado problemáticas generadas por una trama urbana que resultaba insuficiente a las nuevas necesidades. Las viviendas colectivas, la falta de higiene y de hospitales, sumada a la contaminación de las aguas bebibles y de los comestibles, generaron el terreno propicio para la aparición de una serie de enfermedades. Algunas de ellas exóticas, como el cólera o la fiebre amarilla; y otras propias, como la viruela, el sarampión o las diarreas. ${ }^{6}$

En coincidencia con esa dinámica urbana y sanitaria, se producen otros fenómenos tal vez menos perceptibles que estos, que tienen que ver con la niñez y sus concepciones, los que terminarían influyendo en la diagramación de posteriores políticas públicas dando 
origen a lo que hoy conocemos como sanidad infantil, o a renovadas trazas urbanísticas con espacios pensados para el desarrollo saludable de los más pequeños. Ello fue el resultado de que por entonces los niños de la ciudad de Buenos Aires adquirieron una fuerte definición al diferenciarse del mundo de los adultos y al dotar de atributos propios a la infancia. Diferenciación que implica la constitución de identidades distintas de acuerdo al grupo social que se trate. Para ello, Ciafardo (1992) indaga sobre las características distintivas de sus juegos como en las diversas formas de ocupación de los espacios públicos y privados. En ese contexto, es válido pensar que en función de esas distinciones también se generaron diversas maneras de experimentar la tuberculosis según se tratara de niños pobres, de los sectores medios o de los de la elite. En gran medida porque el concepto de niño enfermo también guardó en su interior diferenciaciones según el rango social del que se tratase, hecho que se materializó en distintas búsquedas de soluciones.

Parte de las soluciones implementadas para el tratamiento del niño pobre y tuberculoso, como las razones que facilitaron la expansión de ese mal, deben ser entendidas a la luz de las formas y de las condiciones de vida que la infancia popular tenía, así como también de las ideas médicas en boga tanto en Europa como en América. Para entonces, el trabajo infantil se encontraba en expansión y competía con la tan difundida escolaridad. Juan Suriano (1990) afirma que trabajo y escolaridad eran casi incompatibles debido al tiempo que insumía cualquier empleo, pocas veces inferior a ocho horas diarias de labor. Esta situación generó que la escuela, como espacio de difusión de preceptos higiénicos y de normas, viera limitado su accionar al quedar un universo de niños - en este caso los trabajadores - fuera de su alcance. Por otro lado, las circunstancias en que se efectuaba el trabajo infantil en particular no eran las mejores. Gabriela Coni, en 1901, elevó a la municipalidad un informe donde describía las precarias condiciones laborales de centenares de mujeres ${ }^{7}$ y niños/as. Algunas resultaban nocivas para su salud y eran provocadoras de enfermedades como la tuberculosis. La fatiga, producto de la exigencia del trabajo a destajo como modalidad más difundida, la falta de higiene y ventilación en los locales se combinaban y, de acuerdo al tipo de producto, podían resultar más o menos nocivas. En el caso de las fábricas de bolsas de arpilleras, la pelusa resultaba dañina para las vías respiratorias: "una niña de diez años me aseguraba ser ella muy fuerte, pues aún no se había enfermado, cuando los más grandes solían volverse tísicos ..." (Coni, 1918). La situación descripta se veía agravada porque gran parte de estos niños vivía precariamente en conventillos o casas similares donde prevalecía el hacinamiento y la miseria. También es probable que se alimentaran mal y que la vida familiar en la estrecha pieza no fuera algo precisamente envidiable (Suriano, 1990).

Largas y pesadas jornadas de trabajo, mala alimentación y precarias viviendas, sumadas a las condiciones poco salubres de las barriadas populares a las que seguramente muchos de ellos pertenecían, explican de alguna manera la expansión de ciertas enfermedades entre estos sectores que resultaron más vulnerables que otros. Porque los niños de clase media y alta también enfermaban de tuberculosis, pero eran los pobres los que estaban más propensos a contraerla y por ende menos preparados para combatirla.

Para 1904, los menores de 16 años que trabajan en la Capital Federal sumaban 7.191 de los cuales 4.820 eran varones y 2.371 eran mujeres (Suriano, 1990, p.264, cuadro 33). A estos hay que sumarle una amplia franja de niños que no asistían a los establecimientos 
escolares ni trabajaban que en la literatura histórica los conocemos como el menor abandonado o el mendigo. Esa infancia, la abandonada o callejera, no padecía los gajes del agotador trabajo, pero soportaba de una manera no menos cruel la miseria. Sin viviendas ni una alimentación adecuada, la calle fue un escenario propicio para el desarrollo de enfermedades o la expansión del contagio.

Los observadores de la época generaron una batería de leyes destinadas a encauzar el desorden que producía la acelerada urbanización con los males no deseados de la modernidad. ${ }^{8}$ Uno de esos males era la niñez enferma moral o físicamente que, en gran parte, estaba compuesta por chicos pobres. Debido a esto, se ideó una combinación de mecanismos destinados a reorientar el comportamiento de los pequeños que pululaban por las calles de Buenos Aires, por un lado para extraer rápidamente del espacio público o familiar a los que se enfermaban y por otro, para controlar la diseminación de la infección.

Por ello, con la llamada Ley de Patronato de Menores (1919) se legitima una práctica muy difundida por entonces para atacar los 'peligros detallados' como es la del encierro del 'niño-menor' (Gonzalez, 2000). Para el caso de la infancia en general, el pensamiento higienista proporcionó una serie de medidas basadas en el cuidado de los recién nacidos (salas cunas, inspección de nodrizas, control de la lactancia materna y mercenaria, hospitales y vacunas) tendientes a evitar la mortalidad pos neonatal.

Sin embargo, y a pesar de que para el período estudiado se ponen en marcha una serie de dispositivos institucionales destinados al cuidado de la primera infancia, las muertes de niños menores de cinco años seguían siendo altas. Para 1900, constituían el 44\% de las defunciones generales, de las cuales las diarreas y enteritis fueron crecientes hasta 1914, momento en el que comienza una notoria pendiente negativa. (Nari, 2004, p.27).

\begin{tabular}{|c|c|c|c|c|}
\hline Causa & 1900 & 1910 & 1920 & 1930 \\
\hline Diarreas y enteritis & 34.8 & 35.7 & 35.6 & 22.9 \\
\hline Sistema nervioso & 18.5 & 12.3 & 11.9 & 7.8 \\
\hline $\begin{array}{l}\text { Infecciosas(sarampión, } \\
\text { escarlatina, coqueluche) }\end{array}$ & 2.7 & 1.5 & 2.0 & 3.8 \\
\hline Tuberculosis & 1.3 & 1.1 & 1.5 & 2.3 \\
\hline
\end{tabular}

Fuente: Mazzeo, 1993, p.86.

En ese descenso jugaron un papel muy importante las obras de infraestructura, la aparición de hospitales y la vacunación como el caso de la viruela - tema en el cual no me detendré porque ha sido abordado en otras publicaciones (Álvarez, 2004). Sin embargo, no ocurrió lo mismo con la tuberculosis, lo que revelaría - según Armus (2000, p. 520) - la poca eficacia de las intervenciones médico clínicas o incluso las de salud pública que convivieron con medidas que a pesar de beneficiosas, como la vacuna BCG, el retiro de bebés de madres enfermas, etc., no alcanzaron a modificar los guarismo expresados en el cuadro precedente, por lo menos hasta los años 1930. 
El impacto de esta enfermedad en las estadísticas de mortalidad infantil, a lo largo del período en cuestión, fue significativamente menor que el de las enfermedades gastrointestinales e inclusive que las infecciosas. A pesar de ello, despertó la preocupación y atención de las autoridades, en principio, ocupándose de los llamados niños débiles, propensos a enfermar y, luego, de los que ya padecían ese mal. Sus acciones se orientaron hacia los niños de los sectores populares para los que destinaron diversas iniciativas, de acuerdo a si fueran o no huérfanos.

En el caso que nos ocupa fue el primer Centro de Salud que tuvo la Sociedad de Beneficencia de la Capital Federal, en las afueras de la ciudad (a 400Km de Buenos Aires) y guardó hasta avanzada la década del 1920 el doble carácter de estar destinado a niños débiles y a tuberculosos, pasando luego a convertirse en sanatorio exclusivamente.

\section{Los marcos institucionales generados para los niños enfermos pobres}

La Sociedad de Beneficencia de Capital Federal fue una organización benéfica que reunió a las mujeres de la oligarquía argentina desde principios del siglo XIX. Sus primeras acciones estuvieron guiadas por dos fines primordiales: la dirección y administración de escuelas de niñas y la dirección e inspección de la Casa de Expósitos, el Hospital de Mujeres y todo otro establecimiento público que se creara para asistir a mujeres y niños, como fueron el Asilo Marítimo y el Solarium.

Dichas instituciones deben ser comprendidas en un marco caracterizado por la incidencia que las enfermedades infectocontagiosas tuvieron hasta las primeras décadas del siglo $\mathrm{XX}^{9}$, circunstancia que generó la preocupación de médicos y gobernantes de la ciudad de Buenos Aires por fundar nuevos establecimientos hospitalarios tanto en Capital Federal como en otros lugares de la República. ${ }^{10}$ Con ello, se pretendía lograr simultáneamente una mejor atención y evitar las grandes aglomeraciones de enfermos en los pocos hospitales de la capital. En este sentido, se pueden mencionar propuestas similares a partir de la experiencia de Peter Dettweiler quién inauguró, en 1876, un famoso Sanatorio en Falkenstein (Alemania). El establecimiento otorgaba importancia a las curas al aire libre en amplias terrazas abiertas, bien soleadas y protegidas del viento y de la nieve, donde los enfermos permanecían recostados la mayor parte de la jornada. Esta experiencia de los sanatorios se aplicó principalmente en Alemania y Suiza, creándose instituciones basadas en hotelería de lujo. Posteriormente se pensó en crear los denominados sanatorios de pobres.

Los fundamentos para el funcionamiento de ambos tipos de sanatorios eran similares: reposo, cura de aire y alimentación abundante y variada. Debían cumplir con una serie de requisitos arquitectónicos básicos, como la elección idónea del emplazamiento en medio del campo, rodeado de un paisaje armonioso, bien soleado y protegido de los vientos fríos. La construcción de los espacios comunes (galerías, salas, sistemas de evacuación de aguas residuales) debía realizarse de acuerdo a normas internacionales y debía contar con fácil acceso en calles y caminos. Complemento indispensable de las curas al aire libre era la helioterapia, es decir, el tratamiento mediante la exposición directa del cuerpo a la luz del sol. Asociando la helioterapia con la talasoterapia (tratamiento por el clima y baños de 
mar) se consideraba que los resultados eran mejores y este fue el fundamento para la creación de los Sanatorios Marítimos. ${ }^{11}$

En este contexto, el doctor Emilio Coni, en el año 1892, proponía la creación de un Hospicio y Asilo Marítimo en Mar del Plata para establecer una institución que atendiese a los niños débiles atacados de linfatismo ${ }^{12}$ y escrofulosis ${ }^{13}$, entre otras enfermedades. Además, consideraba que al buen clima del lugar se sumaría la posibilidad de realizar baños de mar como un tratamiento eficaz para los niños convalecientes (Coni, 1895, p.15). Si bien la propuesta fue desalentada por el Patronato de la Infancia, no sucedió lo mismo con la Sociedad de Beneficencia de la Capital que inmediatamente puso en marcha el proyecto.

$\mathrm{Al}$ igual que en la experiencia europea, se restauró un viejo hotel ubicado en las afueras del entonces poblado de Mar del Plata ${ }^{14}$ y en el año 1893 se creaba el Hospital y Asilo Marítimo.$^{15}$ La atención de los niños iba a estar a cargo de la Congregación de las Hermanas Hijas de María del Huerto. La idea que subyacía a este emprendimiento era la de "establecer un albergue a orillas del mar con el doble fin de arrancar a la miseria y a la muerte a un sinnúmero de niños, ora fortaleciendo sus débiles organismos, ora corrigiendo defectos físicos ha sido y es la preocupación constante de la Sociedad de Beneficencia al fundar y sostener este Asilo que hoy funciona prestando inapreciables servicios a los desheredados de la fortuna" (Memoria del Hospital..., 1906, p.323). En función de esto, y a pesar que el pedido original había sido motorizado por la expansión de la tuberculosis infantil, en esta primera etapa convivieron niños anémicos o convalecientes con los que estaban afectados por el bacilo tuberculoso. Este doble carácter hizo que el aislamiento en ciertas oportunidades resultara efímero frente a la virulencia del contagio potenciado por la "estrechez de los locales y la poca extensión que ocupa todo el establecimiento" (Memoria de la Sociedad..., 1916, p.388).

La primera organización contaba con dos secciones principales: la colonia de niños débiles y la sección de sanatorio propiamente dicha. La colonia estaba instalada en un pabellón, con su propio patio, donde se alojaban niños expósitos débiles con la precaución de mantenerlos aislados de los enfermos. Límite que fue reiteradamente violado, produciéndose una "mezcla entre niños ya tuberculizados y niños débiles" (Acta del Consejo..., exp. 7988, feb. 1923). Desde su inauguración, en 1893, los enfermos eran sometidos a tratamientos específicos, controlados por especialistas marplatenses hasta que, en abril de 1900, se incorporó el doctor Héctor Jara, ex interno del hospital Rivadavia. ${ }^{16}$ La incorporación de profesionales se acentuó en la década del 1910, con lo que la atención dejó de brindarse sólo en verano para mantenerse durante todo el año.

De esta manera, en 1915, y a pesar de que en sus memorias el entonces director H. Jara consignara que la institución en cuestión pasaba a ser transformada en Sanatorio Marítimo, trayendo con ello aparejado un cambio en su carácter de asilo y destinándolo pura y exclusivamente a la asistencia de niños afectados por tuberculosis quirúrgicas en sus más variadas localizaciones, esta iniciativa no fue avalada por las Damas de la Sociedad de Beneficencia. La colonia siguió funcionando y lo que se logró fue que al Sanatorio se lo orientara hacia el estudio de las afecciones articulares y ganglionares tuberculosas. La tensión entre los médicos y las Damas de Beneficencia continuó hasta que en 1923 los profesionales 
insistieron en suprimir la colonia, aunque sólo lograron su reducción (Acta del Consejo..., 8 ago. 1923). Este hecho hizo que el afluente de niños siguiera siendo, para el período bajo estudios, tanto de huérfanos como de enfermos. ${ }^{17}$ Recién en 1928, el Consejo Directivo de las Damas de Beneficencia encomienda a la señora Ana de Ortiz Basualdo estudiar la mejor forma de establecer el aislamiento de los niños expósitos de los enfermos (Acta del Consejo..., 27 nov. 1928) y frente a la respuesta de ésta acerca de que "El aislamiento de los niños débiles en la Colonia de Vacaciones del Sanatorio Marítimo será imposible ..." (Acta del Consejo..., 7 dic. 1928), recién entonces es suprimida. Es decir, la colonia dejó de funcionar cuando lo dictaminaron las 'damas', no cuando lo solicitaron los médicos. Esta cuestión señala varios problemas: las tensiones entre los médicos y las damas de beneficencia, la ausencia de la voz del Estado y, por sobre todo, cómo la institucionalización de la tuberculosis no evitaba necesariamente el contagio.

\section{Ser niño y tuberculoso en la Argentina de principios del siglo XX}

La niñez tuberculosa institucionalizada formó parte de la misma construcción sociocultural que operó sobre la infancia abandonada, no escolarizada o 'delincuente' que se consolidó desde fines del siglo XIX. Tanto el Asilo como el Solarium, respondieron a ese universo de conceptos que estaban imbuidos por las preocupaciones en torno a la constitución de una raza fuerte y sana, aunque tuvo sus especificidades y diferencias con respecto a los orfanatos y al mercado laboral del menor.

$\mathrm{Al}$ igual que el proceso europeo, en líneas generales, podríamos afirmar que con el conocimiento adquirido a través de los años y la mejor comprensión de la patología, se planteó desde principio de siglo la necesidad de cortar el contagio, protegiendo a niños, jóvenes y personas no enfermas, recluyendo a los pacientes en hospitales y sanatorios por largos períodos.

En el caso de las instituciones analizadas, la complejidad radica en que la mayoría de sus pacientes eran niños huérfanos derivados de la Casa Expósitos ${ }^{18}$, de los cuales no todos eran tuberculosos, a pesar de que la función del aislamiento marítimo era especialmente orientada a ese mal. En este sentido, en 1893, el médico y director de esa institución, Juan Bosch, reclamaba la creación de este tipo de establecimientos ${ }^{19}$, fundamentando "el número siempre creciente de niños escrofulosos" como también de un número más pequeño de atacados, de conjuntivitis - entre otras enfermedades -, circunstancia que impedía que esos pequeños fueran reubicados en nuevos hogares o en otros establecimientos, agregando que muchos de ellos se curaban, "sin más tratamiento que el mejoramiento del medio en que se les coloca ..." (Bosch, 17 ene. 1893).

La cita precedente deja al descubierto que las motivaciones que llevaron a la creación del Asilo Marítimo no sólo fueron impulsadas por el clima médico de época, sino también por las necesidades que manifestaron otros establecimientos dependientes de la Sociedad de Beneficencia que albergaban un universo de niños que además de abandono padecían de algunas enfermedades producto del contagio contraído en la misma Casa Expósito.

Resulta claro que la constitución física de estos niños era deficiente, si bien con las fuentes disponibles casi resulta imposible - por ahora - diferenciar cuan culpable eran 
las condiciones internas de la Casa Expósitos del estado físico de los niños abandonados que llegaron a la institución con meses de vida de aquellos que lo hicieron previo a pasar situaciones de extrema pobreza. A pesar de esa limitación, podemos sostener que el contagio era una constante y que muchos de esos huérfanos contrajeron la tuberculosis en el marco de ese espacio institucional y no en la 'peligrosa calle' de la cual provenían y en donde, seguramente, no hubieran estado a salvo del mal. En tal sentido, en un primer momento, el Asilo Marítimo era visto como un mejoramiento del medio, pero no sólo del urbano como tradicionalmente lo hemos interpretado sino del institucional. Hecho que marca o pone en discusión las condiciones sanitarias en las que vivían estos huérfanos institucionalizados que, a pesar de esa situación de 'protección', no estaban aislados de los males adjudicados a la pobreza y al abandono 'moral' o higiénico.

En el caso de los pacientes derivados del Hospital de Niños, por lo menos en los primeros años, se visualizan algunos matices en relación al trato que hacia el interior del Asilo Marítimo recibieron. Si bien, igualmente, provenían de estratos sociales populares, no eran huérfanos. Esta puede ser una razón para que las condiciones habitacionales destinadas a estos enfermos fueran mejores que la de los niños expósitos. De hecho, por ejemplo, las condiciones higiénicas de la enfermería ocupada por los niños de la Casa Expósitos es descripta como una pieza pequeña, oscura, mal aireada y hasta húmeda, ubicada en la zona inmediata a los retretes "cuyas emanaciones repugnantes impregnaban la atmósfera encerrada de las galerías que las circundan y por el cúmulo de camas que llegan a once casi siempre ocupadas de día e invariablemente de noche ..." (Jara, 2 ago. 1900, fl.145). Circunstancia que empezaba a ser superada en 1908 con la construcción de un pabellón para baños, enfermería, etc. (Memoria de la Sociedad..., 1909).

Lo anteriormente expresado es significativo porque muestra formas distintas de 'padecer' la enfermedad dentro de un mismo segmento social que se materializa a la hora de distribuir los espacios para unos y otros, donde la desventaja seguía estando del lado de los expósitos, posiblemente porque en el caso de los enfermos del Hospital de Niños, sus padres muchas veces protestaron o retiraron a sus hijos del establecimiento como se puede rastrear a través de las historias clínicas donde quedaban consignados las decisiones de los padres respecto del tratamiento brindado a sus hijos. Es decir, la visibilidad - aunque a la distancia - del progenitor marcó matices de beneficios, en un universo caracterizado por la ausencia de estos últimos. ${ }^{20}$

A pesar de estos 'matices de beneficios', a la hora de enfermar no había grandes diferencias, aunque de hecho la mayor cantidad de afectados por conjuntivitis granulosa eran en su totalidad niños expósitos que además en varios casos compartían coxalgia tuberculosis, cerebro sífilis, artritis tuberculosa en rodillas, mal de patt dorsal ${ }^{21}$, tuberculosis de cuello o raquitismo con los enfermos que provenían del Hospital de Niños (Volumen de Cartas..., libro 1, fl.145, 12 ago. 1900).

Sin embargo, en relación con los expósitos, son estos hijos de obreros pertenecientes a los sectores populares humildes los que en peor estado nutricional arriban al Asilo Marítimo. Resulta claro que en los expósitos, la institucionalización les jugaba a favor en comparación con los otros pequeños. Niños anémicos y raquíticos eran básicamente las derivaciones del Hospital de Niños, los que varias veces llegaron al Marítimo "en estado deplorable" y 
fueron responsabilizados por ello, de las variaciones en los niveles de mortalidad de la institución marplatense (Memoria del Hospital..., 1910, p.324).

Lo expresado hasta el momento es un elemento que contribuye a pensar las condiciones de salud/enfermedad de la infancia popular a principios del siglo XIX, brindando una visión un poco más compleja en torno al problema de la infancia pobre. Si bien estas instituciones fueron pensadas para ellos, al igual que algunas educativas, estaban precedidas por las ideas de aislamiento, del estricto seguimiento clínico antropométrico, del alejamiento de estos pacientes de su espacio familiar o barrial que era interpretado como nocivo. Explicar estas instituciones sólo como una instancia de disciplina físico y moral - en términos de Foucault -, al igual como han sido interpretadas otras de la época, es simplificar su verdadero funcionamiento.

Hacia el interior del Marítimo, la relación paciente-institución (Asilo/niño) no era unidireccional ni se basaba en una lógica de imposiciones impuestas desde la conducción de la Sociedad de Beneficencia o en el plano local por el Asilo. En principio, los pacientes derivados del Hospital de Niños debían contar con la aprobación de sus padres o tutores, con lo cual la 'institucionalización' de esos infantes era un acto consentido entre las partes padres-institución.

Además, a diferencia de los expósitos, los niños procedentes del Hospital de Niños, por su estado físico, fueron los que mayor tiempo pasaron en la institución por prescripción de los médicos y recomendación de las mismas inspectoras. Este punto conlleva una tensión implícita relativa a que esos niños no regresaban a un espacio institucionalizado como los expósitos, sino a sus hogares. Esta situación podría ser uno de los factores por el cual se prolongaba su estadía, si lo pensamos en función del discurso dominante que apuntaba a sacar a "esos menores de la calle" o de los espacios 'nocivos'. Sin embargo, el aplazamiento de la estancia en la mayoría de los casos tenía un fundamento clínico, puesto que si bien

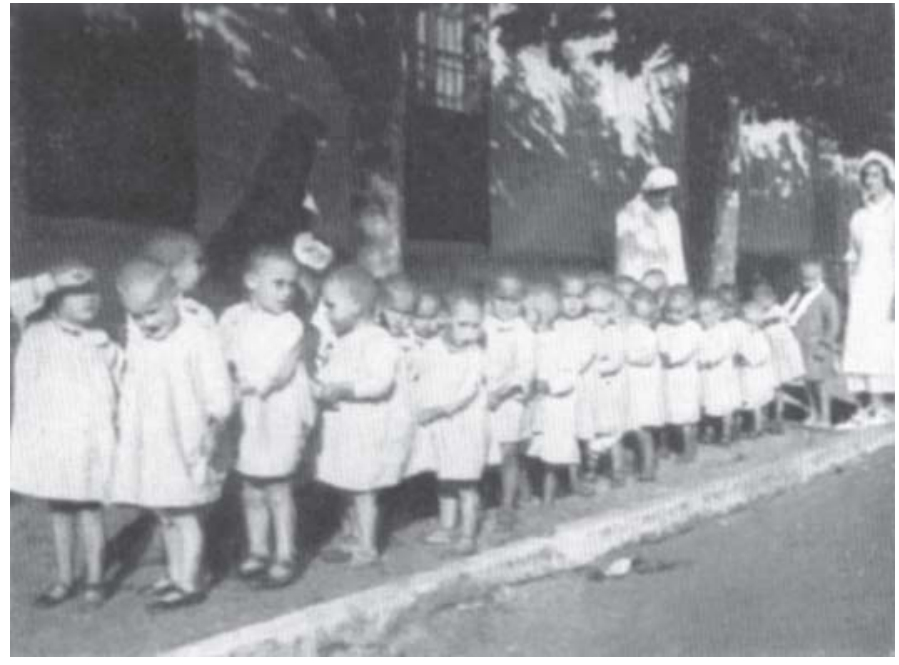

"Un grupo de niños debiles de la colonia de vacaciones". Fondo Sociedad de Beneficencia y Asistencia Social Sanatorio Marítimo, legajo 99, 1927. (Archivo General de la Nación)

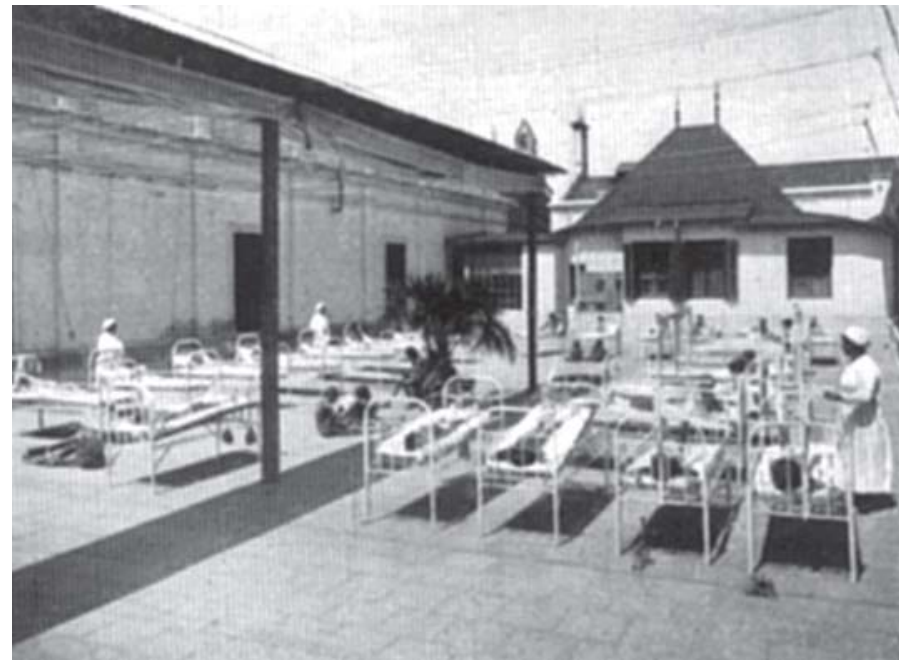

"Sesión de helioterapia en uno de los patios. Para los niños que no pueden ir a la playa". Fondo Sociedad de Beneficencia y Asistencia Social Sanatorio Marítimo, legajo 99, 1927. (Archivo General de la Nación) 
muchos de estos pacientes no eran tuberculosos, el estado de raquitismo con el que habían ingresado, llevaba a especular que, a pesar de su aumento de peso y de glóbulos rojos, eran todavía propensos a contraer la enfermedad. Por ello, en algunas de las fundamentaciones que justifican la extensión de la estadía, se recurre al dicho "más vale prevenir que curar" (Memoria del Hospital..., 1910, p.360).

Por otro lado, exceder el tiempo previsto para la internación implicaba la autorización del progenitor, que no siempre estaba de acuerdo, como en el caso de María E. Peláez que ingresó en abril con diagnóstico de 'sacro coxalga' (artritis causada por infección de cadera, generalmente de origen tuberculoso) y cinco meses más tarde se consigna que "la familia se ha llevado a la paciente a pesar de que no está en condiciones de recibir el alta ..." (Historia clinica..., 1923). Había una negociación entre padres e institución, seguramente lenta y dilatada, dado las condiciones sociales de éstos a quienes se les hacía difícil trasladarse $400 \mathrm{~km}$ a visitar a sus hijos y, por ende, la comunicación era vía indirecta a través del Hospital de Niños que estaba en la ciudad de Buenos Aires. Pero, lo concreto es que en este como en otros casos prevaleció la opinión de los padres por sobre la de los médicos y por sobre la institución, lo que señala los límites a los que el accionar cotidiano estaba sometido, aunque el discurso dominante se orientara a alimentar una serie de imposiciones que consideraba indispensable para el cuidado de la niñez.

Otra cuestión no menos importante a señalar es que, en el plano interno, no hay denominaciones diferenciales para los niños huérfanos de los que no lo eran. Si bien hasta mediados de la década del 1910 tenían salas diferentes, y en los primeros años algunas - no todas - condiciones edilicias diferenciadas (tema al que ya hicimos referencia), la tendencia en las construcciones fue hacia la uniformidad y la equidad en las prestaciones, no importaba si eran niños huérfanos o no. Hacia el interior todos eran los mismos: 'enfermitos', 'niñas enfermas', 'niños enfermos', 'débiles', 'anémicos', 'población infantil'.

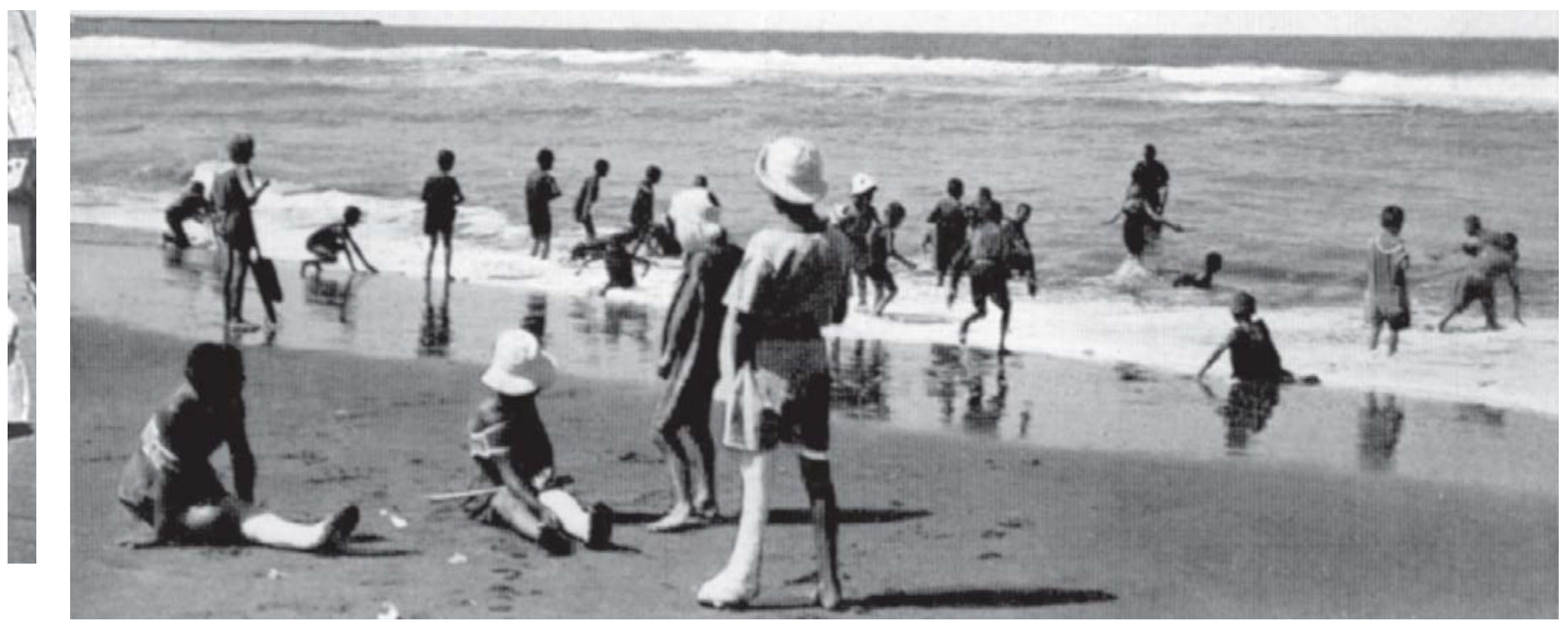

"Durante el baño en el mar, los niños son acompañados por un experto...." Fondo Sociedad de Beneficencia y Asistencia Social Sanatorio Marítimo, legajo 99, 1927. (Archivo General de la Nación) 
No se visualiza en este accionar institucional acotado, lo señalado por Carolina Zapiola (2007) en relación al macro proceso donde por esta época se comenzó a estructurar un discurso por parte de algunos legisladores en base a una antinomia 'niño' (contenidos en los marcos de las familias, la escuela y muchas veces el trabajo) versus 'menores' (niños y jóvenes, pobres, abandonados, delincuentes huérfanos y/o viciosos). Estas concepciones, que han sido también señaladas por otros autores al caracterizar lo que sería el discurso de la época en el seno de instituciones como la acá estudiada, no se presentan de manera directa. Es más, pareciera prevalecer una visión compasiva y hasta humanitaria por sobre la diferenciación que en el plano simbólico se realizaba entre 'niños' y 'menores'. La situación de debilidad estructural pareció borrar las diferencias hacia el adentro de estos establecimientos. Tal vez porque quienes escribieron las Memorias Institucionales (fuentes documentales de primera referencia en este trabajo) no pertenecían ni a las elites políticas ni intelectuales. Fueron médicos como Héctor Jara que asumió la dirección del establecimiento hacia 1900 por un sueldo mínimo, habitación y comida, y permaneció en ese estado por décadas, circunstancia que debe haber contribuido a describir la realidad interior con las únicas herramientas que tenía: las vivencias cotidianas, utilización de la terminología propia de Asilo, con sus denominaciones y connotaciones sin estar cruzado por las variables propias de quien tiene en sus manos parte del destino de una nación como son los legisladores, políticos y funcionarios.

\section{Consideraciones finales}

A lo largo del texto se hacen presentes varias cuestiones. Una de ellas tiene que ver con las características que asumió la institucionalización de la tuberculosis infantil, donde, a pesar de que se recurre al aislamiento, el contagio fue moneda corriente en un espacio donde los niños sanos y enfermos muchas veces se mezclaban. No resultando claro hasta el momento cómo se experimentaba el 'miedo al contagio', puesto que eran constantes los pedidos para pasar estadías de niños o de niñas provenientes de otras instituciones benéficas de Buenos Aires que no eran tuberculosos. Más evidente resulta el hecho de que, a pesar de que existía por parte del discurso dominante una valoración negativa de las familias de donde provenían estos pequeños, cuya actuación desdibujada se describía como despreocupada y abandonada, la mirada hacia el interior de esta institución muestra que estos padres jugaban un papel muy significativo que señala los límites en el accionar de los médicos, de la Sociedad de Beneficencia, como del Hospital. Esta visibilidad de los padres aporta un condimento aún no tenido en cuenta a la hora de contemplar la cuestión social de la infancia enferma y su institucionalización.

Otra cuestión, que dejaremos planteada para un futuro trabajo, tiene que ver con el hecho de que los 'niños del Marítimo', además de experimentar el contacto con la medicina profesional, con la buena alimentación y ropa limpia, tuvieron también la posibilidad de acceder a los juguetes por primera vez. Los juegos en la arena, los 'juguetes' que recibían en donación, las 'hamacas' y el 'teatrito de títeres' ponen en evidencia la voluntad de brindar, por quienes estaban a cargo de la institución, herramientas de integración social, como de 
facilitar o de equiparar ciertos beneficios que gozaban los niños pertenecientes a los sectores medios y altos de esa sociedad.

Esta idea de una integración social por medio del afianzamiento del derecho al juego no había sido contemplado para la niñez popular, por lo cual, para las décadas siguientes, hubo una puesta en valor de ese principio que implicó la materialización de una idea de bienestar bien distinta para la niñez enferma de la que estuvo vigente en décadas anteriores, cuyo énfasis estaba puesto solo en los tratamientos médicos sin prestar atención a la parte lúdica como parte del conjunto de cuestiones que acompañaron a los cuidados recibidos en estos establecimientos.

\section{NOTAS}

* Este artículo es parte de un proyecto financiado por la Agencia de Investigaciones Científicas y Tecnológicas PICTOS/08.

${ }^{1}$ Con relación a la idea de modernidad, recomendamos el texto de François Xavier Guerra,1988.

${ }^{2}$ Este trabajo incorpora una distinción, realizada por Diego Armus (2007), entre niños tuberculosos, pretuberculosos y débiles. Pre-tuberculoso eran los niños a quienes se les identificaba por su débil constitución física, hecho que los convertía en potencial víctima de la enfermedad. Este historiador sostiene que este tema fue catapultado desde comienzos del siglo XX por el creciente uso de los test de tuberculina el cual detectaba a los niños infectados con el bacilo que no estaban enfermos pero que fácilmente podían contraer la enfermedad. Para este grupo de niños fueron pensadas tanto las escuelas para niños débiles como las colonias para niños débiles (p.87).

${ }^{3}$ Sobre la historiografía reciente en América Latina, recomendamos el texto de Birn, 2007.

${ }^{4}$ Ver los trabajos de Nari,1995 y Billorou, 2004.

${ }^{5}$ Los primeros avances fueron realizados desde la creación del Hospital hasta la década del 1920, pudiendo establecer la edad de los niños, su permanencia, el tipo de terapias que se les aplicaba, la derivación de los casos más graves al Solarium, entre otras cuestiones (Molinari, Zuppa, Reynoso, 2006).

${ }^{6}$ Sobre el tema consultar Álvarez, 2004, p.15.

${ }^{7}$ Sobre del tema de la mujer en el mercado laboral, recomendamos Lobato, 2000.

${ }^{8}$ Sobre el tema, ver Zimmernann, 1995.

${ }^{9}$ Los informes referidos solamente a tuberculosis registran una tasa de 14.7 muertos por tuberculosis a cada diez mil habitantes para el año 1914 (Susini, Paso, Zauchinger, 1930, p.13).

${ }^{10}$ Para esto se conformó una comisión que tenía a su cargo el proyecto de ley de ejecución de obras hospitalarias en Capital Federal. El doctor Domingo Cabred, miembro de la misma, había desaconsejado iniciativas que iban a contramano con estas disposiciones y propuestas por la Sociedad. Para ello, se dictó la ley 4953 de 1906 sobre construcción de asilos y hospitales, respondiendo al concepto científico moderno de la descentralización de la asistencia hospitalaria (Informe, $20 \mathrm{sep} .1909$ ).

${ }^{11}$ Con este criterio se instaló en Bussot, Alicante, España, el primer Sanatorio Marítimo en 1897 (Sauret, 2001).

${ }^{12}$ Estado morboso debido al desarrollo excesivo del tejido linfoideo, del timo o tiroides. Generalmente asociado al raquitismo (Capdevila, 1947).

${ }^{13}$ Conjunto de alteraciones de los ganglios linfáticos, de la piel, mucosas, huesos, etc. que reconocen constantemente un origen tuberculoso (Capdevila, 1947).

${ }^{14}$ La Ciudad de Mar del Plata está ubicada geográficamente sobre el Océano Atlántico en el partido General Pueyrredón, al sudeste del Gran Buenos Aires. Para la década del 1880 se había convertido en el Biarritz argentino, puesto que fue escogido por la aristocracia porteña para pasar en allí sus veranos, transformando de esta manera su fisonomía de pueblo rural en un moderno espacio urbano, con una traza arquitectónica europea, grandes casonas, teatros, hoteles que hicieron de este rincón de la provincia de Buenos Aires, un balneario al mejor estilo de los europeos.

${ }^{15}$ Posteriormente pasó a llamarse Sanatorio Marítimo, aunque informalmente se siguió mencionándolo como Hospital y Asilo Marítimo. 
${ }^{16}$ El doctor Héctor Jara aceptó las condiciones ofrecidas de una mensualidad de 150 pesos moneda nacional, comida y habitación en el Asilo (Volumen de Cartas..., libro 1, fl.122, 5 abr. 1900).

17 Sobre el tema de las tensiones entre estos médicos y las damas de la Sociedad de Beneficencia, ver Álvarez, Reynoso, Molinari, 2004.

${ }^{18}$ En 1852, se restablece en la ciudad de Buenos Aires, la Casa de Expósitos que contaba para 1909 con un importante hospital para la asistencia de la primera infancia con capacidad para 300 enfermos: "han sido recogidos desde su fundación hasta el año pasado 42.422 niños expósitos, de los que 35.740 han sido bajo la superintendencia de la Sociedad o sea desde el año 1852 hasta el 31 de diciembre pasado ..." (Memoria de la Sociedad..., 1910, p.10 y ss). Sin embargo, cabe consignar que años más tarde se abrieron los consultorios externos gratuitos a todos los niños pobres que lo necesitaran, aunque no tuvieran el carácter de expósitos.

${ }^{19} \mathrm{El} 1^{\mathrm{o}}$ de enero de 1893, tras una visita de los doctores Juan Bosch y Antonio Arraga, directores de la Casa Expósitos y Hospital de Niños de Buenos Aires, elevaron a la Sociedad de Beneficencia de la Capital Federal un informe en el que manifestaban la conveniencia de fundar un sanatorio marítimo en beneficio de los niños convalecientes de los dos establecimientos (Volumen de cartas..., libro 1, fls.17-20, 17 jul. 1893).

${ }^{20}$ Durante diciembre de 1922 se "contestaron 54 cartas a los padres de los enfermitos que preguntan por el estado de salud de los mismos y tres telegramas con el mismo objeto". Estos informes sobre las cartas respondidas se hacen en forma periódica a lo largo de las memorias (Sociedad de Beneficencia..., exp. 7510).

${ }^{21}$ En esta patología se experimentaba un compromiso del estado general del enfermo, el cual se siente con falta de fuerzas, decaído, con pérdida de apetito y peso. El dolor puede ser cervical, dorsal o lumbar, de acuerdo a la localización, con rigidez de columna y dificultad para la deambular por dolor, cansancio precoz.

\section{REFERENCIAS}

ACTA DEL CONSEJO...

Acta del Consejo Directivo de las Damas de Beneficencia. Sociedad de Beneficencia de la Capital Federal. Legajo 98. (Archivo General de la Nación).

ÁLVAREZ, Adriana.

El reinado y el control de las endemias en la ciudad de Buenos Aires de fines del siglo XIX y principios del XX. In: Álvarez, Adriana; Reynoso, Daniel; Molinari, Irene (Ed.). Historias de salud y enfermedad en la Argentina de los siglos $X I X$ y XX. Buenos Aires: Editorial de la Universidad Nacional de Mar del Plata. p.15-46. 2004.

ALVAREZ, Adriana; REYNOSO, Daniel, MOLINARI, Irene (Ed.).

Historias de enfermedades, salud y medicina, en la Argentina de los siglos XIX y XX. Buenos Aires: Editorial de la Universidad Nacional de Mar del Plata. 2004.

ARIÈS, Philippe.

El niño y la vida familiar en el antiguo régimen. Madrid: Taurus. 1987.

ARMUS, Diego.

La ciudad impura: salud, tuberculosis y cultura en Buenos Aires, 1870-1950. Buenos Aires: Edhasa. 2007.

ARMUS, Diego.

Historias de enfermos tuberculosos que protestan, Argentina, 1920/1940. Buenos.Aires: Lugar. 2005.
ARMUS, Diego.

El descubrimiento de la enfermedad como problema social. In: Lobato, Mirta (Ed.). Nueva historia argentina: el progreso la modernización y sus límites (1880/1916), t.5. Buenos.Aires: Sudamericana. p.507-552. 2000.

ARMUS, Diego.

Salud y anarquismo: la tuberculosis en el discurso libertario argentino, 1890/1940. In: Lobato, Mirta (Ed.). Política, médicos y enfermedades. Buenos Aires: Biblos-UNMDP. p.91-116. 1996.

BARRANCOS, Dora.

Inclusión exclusión: historia con mujeres. Buenos Aires: Fondo de Cultura Económica. 2001.

BARRANCOS, Dora.

Moral sexual, sexualidad y mujeres trabajadoras en el período de entreguerras. In: Devoto, Fernando; Madero, Marta (Ed.). Historia de la vida privada en la Argentina. t.3: La Argentina entre multitudes y soledades: de los años treinta a la actualidad. Buenos Aires: Taurus. p.199-225. 1999.

BILLOROU, María José.

Esta sociedad ha llegado en un momento oportuno: nació aunando pensamiento y ejecución - la creación de la Sociedad de Puericultura de Buenos Aries. In: Álvarez, Adriana; Reynoso, Daniel; Molinari, Irene (Ed.). Historias de salud y enfermedad en la Argentina de 
los siglos XIX y XX. Buenos Aires: Editorial de la Universidad Nacional de Mar del Plata. p.187-208. 2004.

BIRN, Anne-Emanuelle.

Saúde infantil na América Latina: perspectivas historiográficas e desafios. História, Ciências, Saúde - Manguinhos, Rio de Janeiro, v.14, n.3, p.677-708. 2007.

\section{BONAUDO, Marta.}

Cuando las tuteladas tutelan y participan: la Sociedad de Damas de Caridad (1869-1894). Signos Históricos, México DF, n.15, p.70-97. 2006.

BOSCH, Juan.

Carta a la presidenta de la Sociedad de Beneficencia, Etelvina C. de Sale. Sociedad de Beneficencia de la Capital Federal. Índice de asuntos contenidos en el volumen n.1, fl.17. (Archivo General de la Nación). 17 ene. 1893.

CAPDEVILA, Casas E.

Diccionario terminológico de ciencias médicas. Barcelona: Salvat. 1947.

CARBONETTI, Adrián.

Beneficencia y tuberculosis: tensiones y conflictos en torno a la formación del sistema asistencial en la ciudad de Córdoba, 1914/1947. In: Álvarez, Adriana; Reynoso, Daniel; Molinari, Irene (Ed.). Historias de enfermedades, salud y medicina en la Argentina de los siglos $X I X-X X$. Buenos Aires: Editorial de la Universidad Nacional de Mar del Plata. 2004.

CIAFARDO, Eduardo.

Los niños en la ciudad de Buenos Aires

(1890-1910). Buenos Aires: Ceal. 1992.

CONI, Emilio.

Contribución a la historia de la legislación obrera argentina. Boletín Mensual del Museo Social Argentino, Buenos Aires, año 7, n.75-80, 81-84. 1918.

CONI, Emilio.

Hospicio y asilo marítimo: proyecto de creación en Mar del Plata. Revista de Higiene Infantil, Buenos Aires, n.1. 1895.

DI LISCIA, María Silvia.

Colonias y escuelas de niños débiles. Los instrumentos higiénicos para la eugenesia: primera mitad del siglo XX en Argentina. In: Di Liscia, M. Silvia; Bohoslavsky, Ernesto. Instituciones y formas de control social en América Latina, 1840-1940. Buenos Aires: Prometeo. 2005.

GONZÁLEZ, Fabio Alberto.

Niñez y beneficencia: un acercamiento a los discursos y las estrategias disciplinarias en torno a los niños abandonados en Buenos Aires de principios de siglo XX (1900-1930). In: Moreno,
José Luis (Comp.). La política social antes de la política social. Buenos Aires: Trama. p.129-204. 2000

GUERRA, François Xavier.

México: del antiguo régimen a la revolución. México DF: FCE. 1988.

GUY, Donna.

La 'verdadera historia' de la Sociedad de Beneficencia. In: Moreno, José Luis (Comp.). La política social antes de la política social. Buenos Aires: Trama. p.321-341. 2000.

GUY, Donna.

Niños abandonados en Buenos Aires (18801914) y el desarrollo del concepto de madre. In: Fletcher, Lea (Comp.). Mujeres y cultura en la Argentina del siglo XIX. Buenos Aires: Feminaria. p.217-227. 1994.

\section{INFORME.}

Nota del presidente de la comisión al sr. ministro del 20 de septiembre de 1909. Legajo 98, expediente 7382. (Archivo General de la Nación). 20 sep. 1909.

JARA, Hector.

Carta en Memoria del Primer Trimestre del año 1900. Hospital Asilo Marítimo, Sociedad de Beneficencia de la Capital Federal, Índice de asuntos contenidos en el volumen n.1, fl.145. (Archivo General de la Nación). 2 ago. 1900.

\section{HISTORIA CLINICA...}

Historia Clínica n.802, de María E. Peláez, 12 años de edad, procedente del Hospital de Niños. Libro de historias clínicas, perteneciente a la Sala de Mujeres del Sanatorio Marítimo. (Biblioteca del Instituto Nacional de Epidemiologia). 1923.

LIONETTI, Lucía.

La escuela pública y su acción sobre la infancia en riesgo en la Argentina de comienzos del siglo $\mathrm{XX}$ : del 'laboratorio para enseñar' al 'laboratorio de las políticas eugenistas'. Presentado en Jornada Historia de la Infancia en Argentina, 1880-1960: Enfoques, Problemas y Perspectivas, 18 nov. 2008. Buenos Aires. 2008.

\section{LOBATO, Mirta Zaida.}

Entre la protección y la exclusión: discurso maternal y protección de la mujer obrera argentina, 1890-1934. In: Suriano, Juan (Comp.). La cuestión social en Argentina, 1870-1943. Buenos Aires: La Colmena. p.245- 276. 2000.

LOBATO, Mirta Zaida (Ed.). Política, médicos y enfermedades: lecturas de historia de la salud en la Argentina. Buenos Aires: Biblos. 1996. 
MAZZEO, Victoria.

Mortalidad infantil en la ciudad de Buenos Aires (1856-1986). Buenos Aires: Ceal. 1993.

\section{MEMORIA DE LA SOCIEDAD...}

Memoria de la Sociedad de Beneficencia de 1915. Buenos Aires: Talleres del Asilo de Huérfanos. p.388. 1916.

\section{MEMORIA DE LA SOCIEDAD...}

Memoria de la Sociedad de Beneficiencia de la Capital Federal de 1909. Buenos Aires: La Semana Médica. p.10 y ss. 1910.

\section{MEMORIA DE LA SOCIEDAD..}

Memoria de la Sociedad de Beneficencia de la Capital Federal de 1908. Memoria del Hospital y Asilo Marítimo. Mar del Plata: Imprenta Malena. p.357. 1909.

MEMORIA DEL HOSPITAL...

Memoria del Hospital y Asilo Marítimo (Mar del Plata - Provincia de Buenos Aires, 1908). En: Sociedad de Beneficencia de la Capital Federal. Memoria de la Sociedad de Beneficencia de la Capital Federal. Buenos Aires: Imprenta Malena. 1910.

MEMORIA DEL HOSPITAL...

Memoria del Hospital y Asilo Marítimo de Mar del Plata. 1906.

MOLINARI, Irene; ZUPPA, Irene; REYNOSO, Daniel.

Mejor curarlos que atenderlos toda la vida: las prácticas cotidianas del Sanatorio Marítimo en la década de 1920. En: Alvarez, Norberto; Rustoyburu, Cecilia; Zuppa, Graciela (Org.). Pasado y presente de la Mar del Plata Social. Mar del Plata: Eudem. p.185-200. 2006.

MORENO, José Luis.

La política antes de la política social: caridad, beneficencia y política social em Buenos Aires, siglos XVII a XX. Buenos Aires: Prometeo. 2000.

NARI, Marcela María.

Políticas de maternidad y maternalismo político. Buenos Aires: Biblos. 2004.

NARI, Marcela María.

La educación de la mujer (o acerca de cómo cocinar y cambiar los pañales a su bebé de manera científica). In: Mora. Revista del Área Interdisciplinaria de Estudios de la Mujer, Buenos Aires, n.1. 1995.

PITA, Valeria Silvina.

Cabellos largos, ideas cortas: las difíciles relaciones entre las mujeres de la Sociedad de Beneficencia de la capital y los médicos porteños, 1880-1905. En: Bravo, Maria Celia; Lozano, Fernanda G.; Pita, Valeria (Comp.). Historia de luchas, resistencias y representaciones: mujeres en la Argentina, siglos XIX y XX. Tucumán: Editorial de la Universidad Nacional de Tucumán. 2007.

PITA, Valeria Silvina. ¿La ciencia o la costura $i$ : pujas entre médicos y matronas por el dominio institucional Buenos Aires, 1880-1903. En: Alvarez, Adriana; Molinari, Irene; Reynoso, Daniel (Ed.). Historias de enfermedades, salud y medicina en la Argentina de los siglos XIX-XX. Mar del Plata: Universidad Nacional de Mar del Plata. p.81-109. 2004.

POLLOCK, Linda.

Los niños olvidados: relaciones entre padres e hijos de 1500 a 1900. México: FCE. 1983.

RUSTÁN, María E.; CARBONETTI, Adrián. El trabajo de niños en contextos urbanos en Argentina: casos ciudad de Córdoba y ciudad de Buenos Aires a principios del siglo XX. Cuadernos de Historia, Serie Población, Córdoba, n.2, p.163-185. 2000.

SAURET, Jesús.

La cura sanatorial de la tuberculosis. Historia de las Enfermedades Emergentes, Barcelona, v.3, n.4, p.199-205. 2001.

\section{SOCIEDAD DE BENEFICENCIA...}

Sociedad de Beneficencia de la Capital Federal. Legajo 7988, expediente 7510. (Archivo General de la Nación).

STONE, Lawrence.

El pasado y el presente. México DF: FCE. 1986.

SURIANO, Juan.

Niños trabajadores: una aproximación al trabajo infantil en la industria porteña de comienzos de siglo. In: Armus, Diego (Comp.). Mundo urbano y cultura popular. Buenos Aires: Sudamericana. p.251-280. 1990.

SUSINI, Miguel; PASO, Juan; ZAUCHINGER, Adela.

La tuberculosis en la Argentina: evolución de la mortalidad. Anales del Departamento Nacional de Higiene, Buenos Aires, v.37, n.2, p.5-54. 1930.

VALLEJOS, Gustavo.

Males y remedios de la ciudad moderna: perspectivas ambientales de la eugenesia, Argentina de entreguerras. Asclepio. Revista de Historia de la Medicina y de la Ciencia, Madrid, v.59, n.1, p.203-238. 2007.

\section{VOLUMEN DE CARTAS..}

Volumen de Cartas y Pedidos para el Hospital y Asilo Marítimo. Sociedad de Beneficencia de la Capital Federal, libro 1. (Archivo General de la Nación). 
La experiencia de ser un 'nino débil y enfermo'...

ZAPIOLA, Carolina.

Niños en las calles: imágenes literarias y representaciones oficiales en la Argentina del Centenario. In: Gaysol, Sandra; Madero, Marta (Ed.). Formas de historia cultural. Buenos Aires: Prometeo. p.305-332. 2007.
ZIMMERMANN, Eduardo.

Los reformistas liberales. Buenos Aires:

Ed. Sudamericana. 1995

$\rightarrow \rightarrow \rightarrow<<<$ 